\title{
Crystal Diffraction Lens for Medical Imaging
}

\author{
Robert K. Smither and D. Eduardo Roa \\ User Program Division, Advanced Photon Source \\ Argonne National Laboratory, * Argonne, IL 60439
}

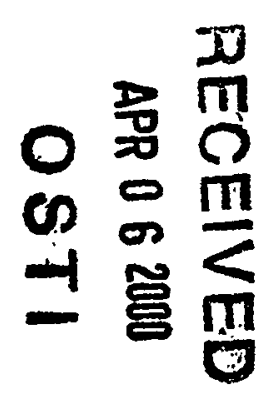

February 2000

\begin{abstract}
The submitted manuscript has been created by
the University of Chicago as Operator of

Argonne National Laboratory (Argonne) under

Contract No. W-31-109-ENG-38 with the U.S.

Department of Energy. The U.S. Govermment

retains for itself, and others acting on its behalf,

a paid-up, nonexclusive, irrevocable worldwide

license in said article to reproduce, prepare

derivative works, distribute copies to the public,

and perform publicly and display publicly, by

or on behalf of the Government.
\end{abstract}

To be presented at the SPIE Medical Imaging 2000 Conference, San Diego, CA, Feb 12-17, 2000, and published in the Proceedings. *This work is supported by the U.S. Department of Energy, Basic Energy Sciences-Materials
Sciences, under contract \#W-31-109-ENG-38. 


\section{DISCLAIMER}

This report was prepared as an account of work sponsored by an agency of the United States Government. Neither the United States Government nor any agency thereof, nor any of their employees, make any warranty, express or implied, or assumes any legal liability or responsibility for the accuracy, completeness, or usefulness of any information, apparatus, product, or process disclosed, or represents that its use would not infringe privately owned rights. Reference herein to any specific commercial product, process, or service by trade name, trademark, manufacturer, or otherwise does not necessarily constitute or imply its endorsement, recommendation, or favoring by the United States Government or any agency thereof. The views and opinions of authors expressed herein do not necessarily state or reflect those of the United States Government or any agency thereof. 


\section{DISCLAIMER}

Portions of this document may be illegible in electronic image products. Images are produced from the best available original document. 


\title{
Crystal diffraction lens for medical imaging
}

\author{
Robert K. Smither* and D. Eduardo Roa \\ Argonne National Laboratory, Argonne IL 60439
}

\begin{abstract}
A crystal diffraction lens for focusing energetic gamma rays has been developed at Argonne National Laboratory for use in medical imaging of radioactivity in the human body. A common method for locating possible cancerous growths in the body is to inject radioactivity into the blood stream of the patient and then look for any concentration of radioactivity that could be associated with the fast growing cancer cells. Often there are borderline indications of possible cancers that could be due to statistical functions in the measured counting rates. In order to determine if these indications are false or real, one must resort to surgical means and take tissue samples in the suspect area. We are developing a system of crystal diffraction lenses that will be incorporated into a 3-D imaging system with better sensitivity (factors of 10 to 100) and better spatial resolution (a few $\mathrm{mm}$ in both vertical and horizontal directions) than most systems presently in use. The use of this new imaging system will allow one to eliminate 90 percent of the false indications and both locate and determine the size of the cancer with $\mathrm{mm}$ precision. The lens consists of 900 single crystals of copper, $4 \mathrm{~mm} \times 4 \mathrm{~mm}$ on a side and $2-4 \mathrm{~mm}$ thick, mounted in 13 concentric rings.
\end{abstract}

Keywords: Crystal Diffraction, Lens, Focusing, Gamma Rays, Medical Imaging, Cancer, Tumor

\section{INTRODUCTION}

A very successful method of locating cancerous tissue in the human body is to inject a biological compound that is readily incorporated in growing cancer cells and that can carry a radioactive nucleus with it, which is also incorporated in the cancer cells. One then performs a partial or full body scan of the patient looking for concentrations of radioactivity above what is incorporated into normal cells. Typically this is done with large NaI detectors, behind a very fine multihole collimator. ${ }^{1-4}$ Some of these systems rotate to obtain a better 3-D image of the cancer. ${ }^{1,5,6}$ Even with the best of these imaging systems, tumors less than $1 \mathrm{~cm}$ in diameter are hard to $\mathrm{se}^{1,7}$ and false tumor indications do occur. Often the only way to be sure is to take a biological sample. The new imaging system ${ }^{8}$ described in this paper is designed to be used after the full body scan to check the validity of these questionable indications of a cancer. No new radioactivity needs to be added to the blood stream since the new system should be able to see the radioactivity already in the body. We estimate that the new imaging system will be 10 to 20 times more sensitive than the full body scans and will be able to eliminate 80 to 90 percent of the false indications. ${ }^{7.8}$ It should also be able to determine the size and location of the tumor with $\mathrm{mm}$ resolution. This will improve the accuracy of any biological sampling and / or surgical removal of the tumor. It may even be possible to combine the imaging system with the sampling system, using the imaging system to accurately position the sampling probe.

\section{NEW IMAGING SYSTEM}

The new imaging system was developed at Argonne National Laboratory. Figure 1 is an artist's view of the proposed new imaging system, employing six of the new crystal diffraction lens systems for sensing radioactivity in the human body. By using more than one lens system that look at a common point from different angles, one can obtain a 3-D image by scanning the source. The large collection area of the lens and the small size of the detector result in a very high signal-to-noise ratio for the system. The detection efficiency of the system remains high for very small sources of $1 \mathrm{~mm}$ and less. The desired goal of this work is to be able to see tumors as small as $2-3 \mathrm{~mm}$ in diameter.

\footnotetext{
*E-mail: rks@aps.anl.gov; Telephone: 630-252-4959; Fax: 630-252-9303
} 


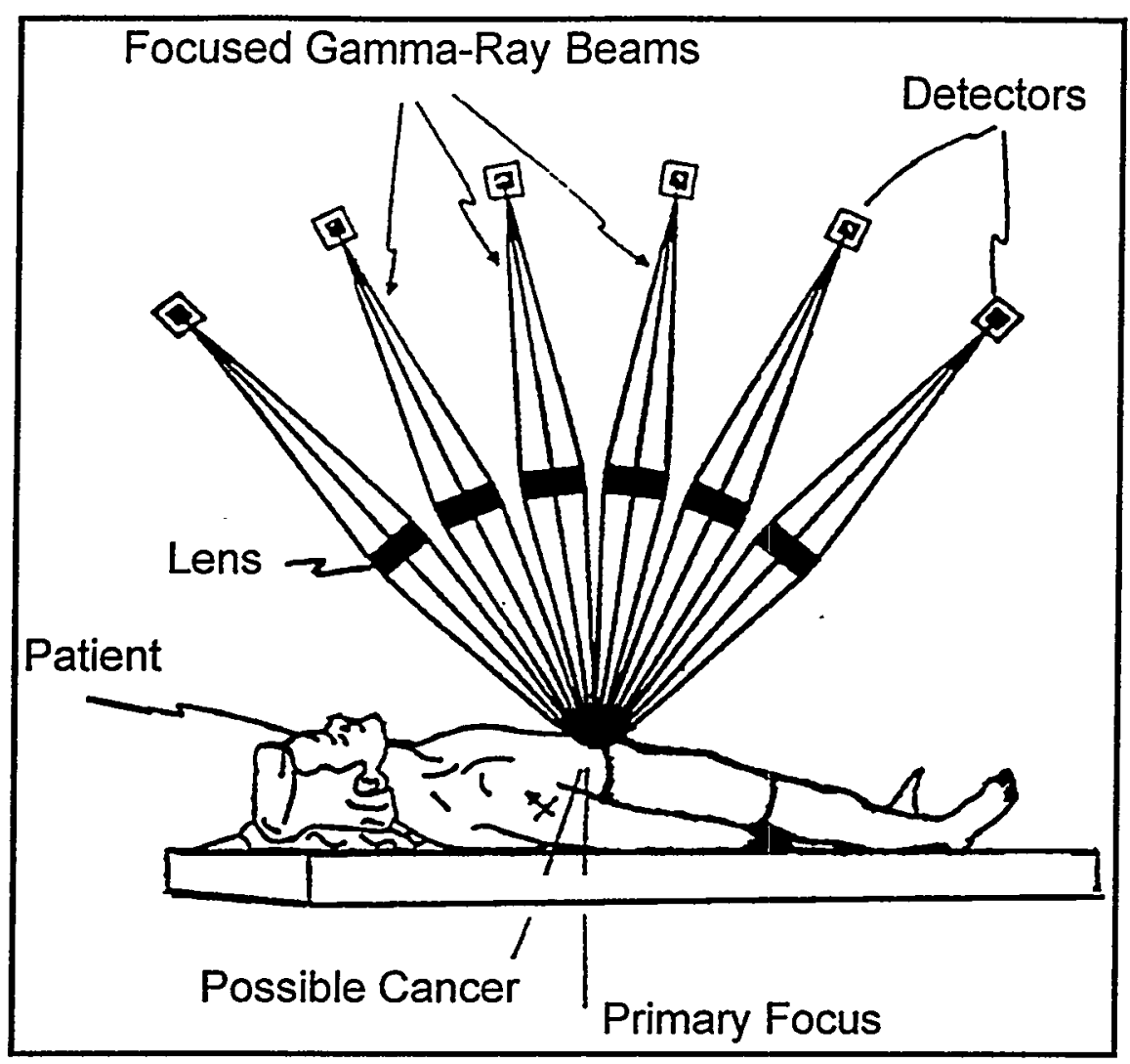

FIGURE 1. Artist's view of the new medical imaging system using six Laue lenses mounted on six different viewing planes.

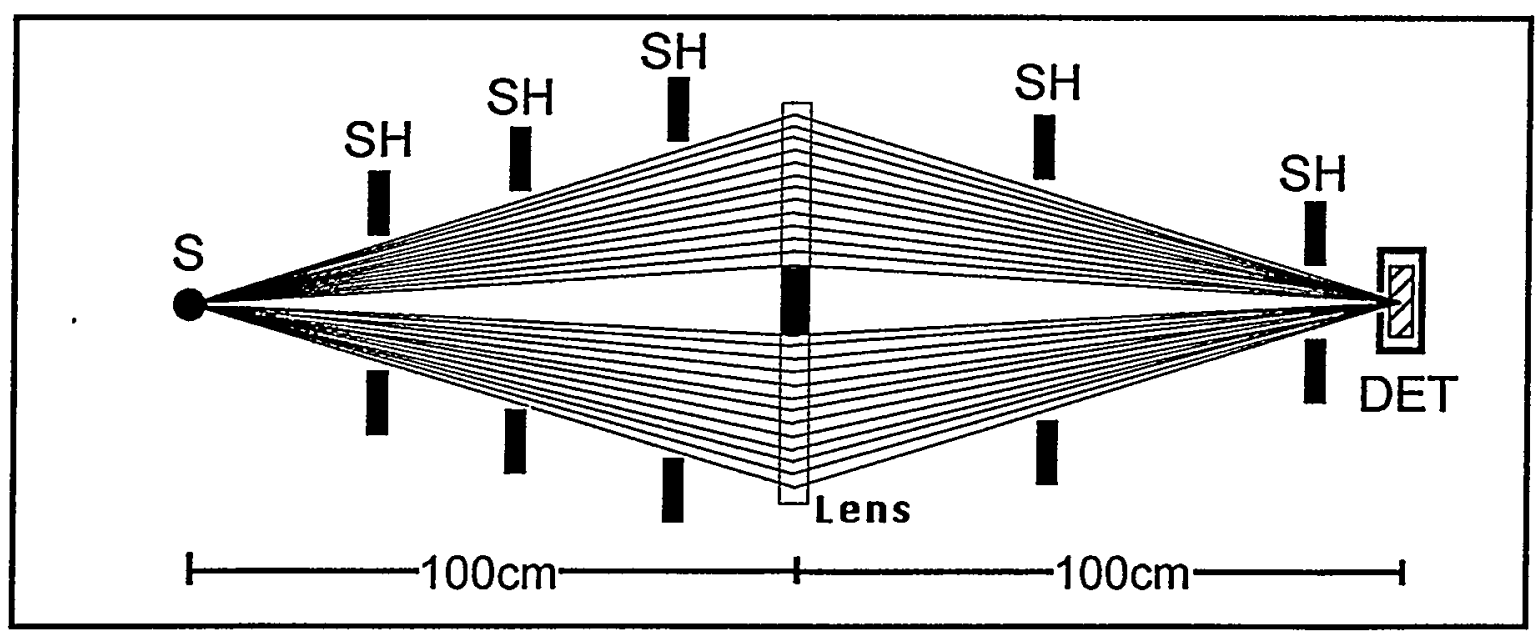

FIGURE 2. Schematic of one Laue type crystal lens focusing system where $\mathrm{S}=$ source, $\mathrm{DET}=$ detector and $\mathrm{SH}=$ collimator shield. 


\subsection{The Individual Lens System}

A schematic of the individual lens system is shown in Figure 2. The system consists of a Laue type crystal diffraction lens mounted about half way between the potential radioactive source and the detector, with appropriate collimator disks located between the source and the lens and between the lens and the detector. These collimator disks are lead plates with a central hole of the appropriate size to let the diffracted beam reach the detector but to prevent any other radiation from the source area from reaching the detector. This lens system can be adjusted to focus a large range of energies by adjusting the distance from the source to the lens and making a similar adjustment in the distance from the lens to the detector. For the Laue-type crystal diffraction lens, in which the gamma rays are diffracted as they pass through the crystals, the focal length of the lens is proportional to the energy of the diffracted gamma ray. In the system described in this paper, the lens is located at twice the focal length distance from both the source and the detector, a geometry that reduces the source-to-detector distance to a minimum. The crystal lens behaves much like a simple convex lens for visible light. The simple relation:

\section{Focal Length $=$ Constant $\times$ Gamma Ray Energy}

holds as long as the sine of the Bragg angle equals the tangent of the Bragg angle. For the energy range that this lens is designed to focus, 100 to $150 \mathrm{keV}$, the Bragg angles are only a few degrees, so this approximation is quite good. The gamma ray of $140.5 \mathrm{keV}$ that one will use in the human tests focuses at a distance of $100 \mathrm{~cm}$, while the $122.0 \mathrm{keV}$ line from ${ }^{57} \mathrm{Co}$ that is used in the lab tests focuses at $87 \mathrm{~cm}$.

\subsection{The Laue Crystal Diffraction Lens}

The Laue crystal diffraction lens consists of 13 concentric rings of copper crystals, $4 \mathrm{~mm} \times 4 \mathrm{~mm}$ and 2-4 $\mathrm{mm}$ thick. Each ring uses a particular set of diffraction planes. The radius of the ring is adjusted so that the diffracted beam is focused on a common focal spot. Figure 3 is a schematic view of the lens from an on-axis position above the lens. The outer diameter of the area covered by the crystals is $14.4 \mathrm{~cm}$. The inner diameter is $3.2 \mathrm{~cm}$. The approximate area of the crystals is $155 \mathrm{~cm}^{2}$. The theoretical diffraction efficiency of the lens (diffracted gamma rays divided by the incident gamma rays) is calculated to be between 3 and 5 percent, for the $140.5 \mathrm{keV}$ line, depending on how well the crystals are aligned and how well the mosaic width of the crystals is controlled. Using the 5 percent value, the efficiency of a singlelens system for a small source at $100 \mathrm{~cm}$ from the lens is $6.2 \times 10^{-5}$. For a six-lens system the combined efficiency would be $3.7 \times 10^{-4}$. A one micro-Curie source gives off $3.7 \times 10^{4}$ gammas per sec. With this source, the count rate in the lens system would be 14 counts per sec. With the combined background count rate of 1.4 counts per sec for the 6 detectors, the signal-to-background ratio would be to 10:1. Thus one would detect the source in a few seconds, and one could expect to detect sources of $1 / 10$ of a microCurie and less in a few tens of seconds. This calculation assumes the use of small NaI detectors in the lens system. It also assumes no absorption of the gamma rays in the body. This absorption will be similar to the absorption as of the gamma rays in 4 to $8 \mathrm{~cm}$ of water where the transmission is 0.56 and 0.30 , respectively.

\subsection{Construction of the Crystal Diffraction Lens}

The rings of crystal in the lens are supported by a thin, 1.5-mm-thick, low-Z material. In Figure 3, the 13 rings cover the whole area of the lens, with no space between crystals or crystal rings. This approach would be very hard to assemble in practice. To avoid this crowding problem and make the assembly easier, every other ring is mounted on a second plate. Thus, rings $1,3,5,7,9,11$, and 13 are mounted on one plate, while rings $2,4,6,8,10$, and 12 are mounted on the second plate. A cross section of the lens showing the alternate crystal rings on the two plates is shown as the lower image in Figure 3 . The original design of the crystal diffraction lens had only 8 rings of crystals, and each ring used a different set of crystalline planes. In the new design for the crystal lens, there are 13 rings that use a total of 9 


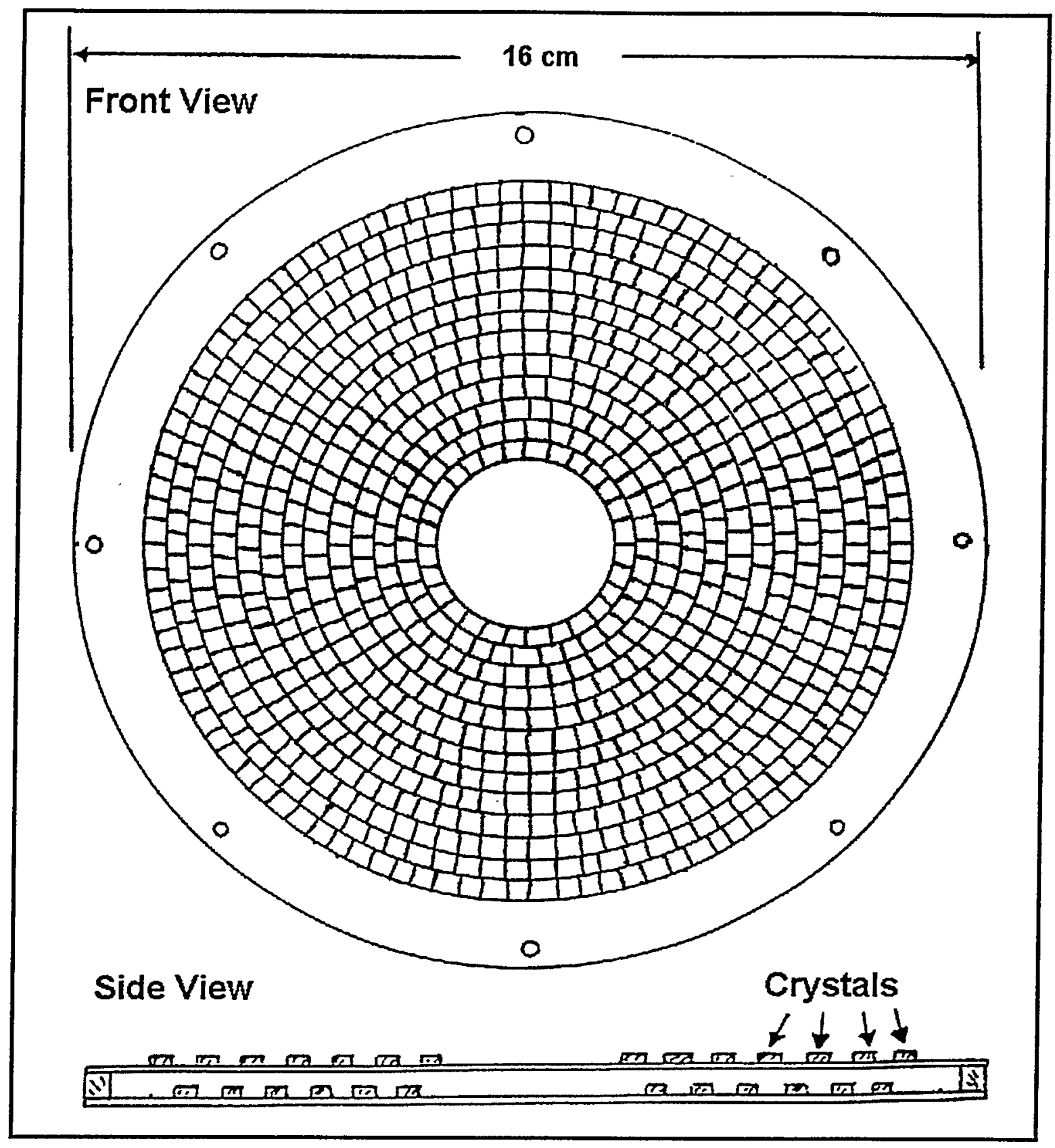

FIGURE 3. The upper image is an on-axis view of the Laue lens from above, showing all 13 concentric rings of crystals. Each crystal is $4 \mathrm{~mm}$ on a side and $2 \mathrm{~mm}$ to $4 \mathrm{~mm}$ thick. The lower image is a side view of the lens, showing the sets of alternate crystal rings mounted on two separate flat plates. 


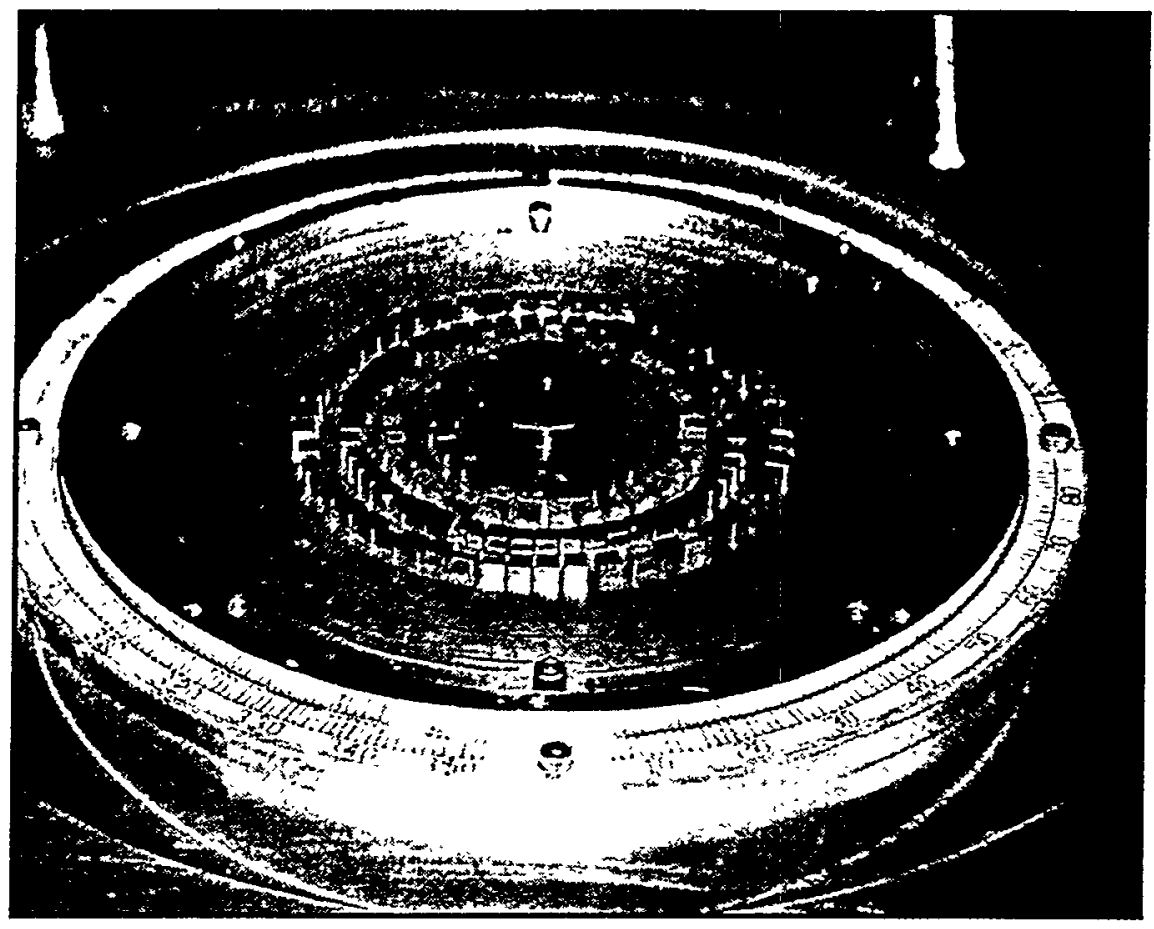

FIGURE 4. The aluminum plate lens frame with crystal rings $1,3,5$ and 7 mounted on it.

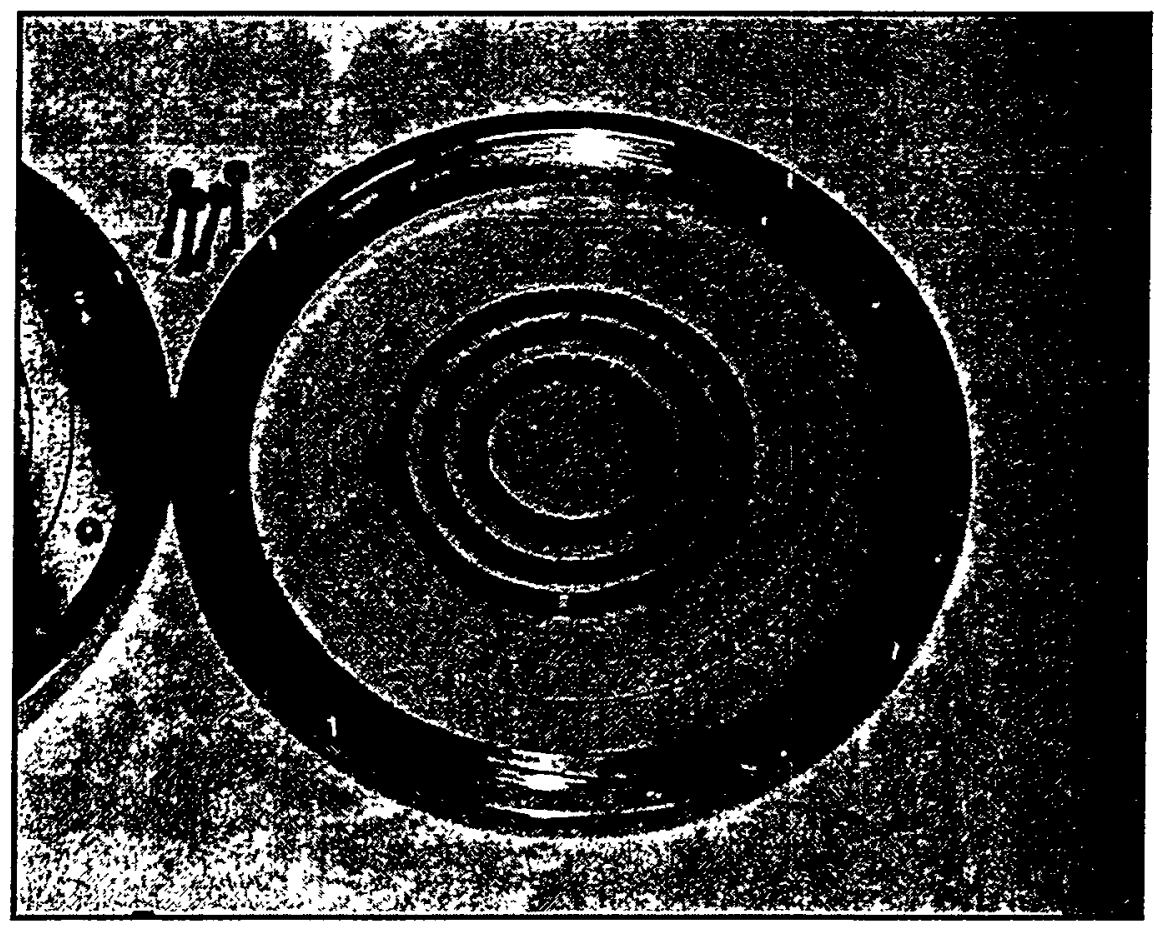

FIGURE 5. View of the quart\% plate lens frame with crystal rings 2, 4 and 6 mounted on it as seen from an on-axis position above the lens. 
crystalline planes. This means that four of the crystalline planes are used twice. In these cases, one of rings is moved in closer to the center of the lens and one of the rings with the same set of crystalline planes is moved out from the center of the lens. In order that the same Bragg angle be maintained for the crystals in both rings, the surface of the crystals are cut at different angles for the two rings. This just compensates for the change in radius. This change increases the diameter of the focal spot, which increases the background count rate by a small amount, but is well worth this increase because it increases the efficiency of the lens by 63 percent. The spatial resolution of the lens remains the same.

As mentioned above, the lens is constructed by mounting rings of crystals on a flat plate (see Figure 3 ). Each crystal in each ring has to be orientated so that the gamma rays from the source are incident on the crystalline planes with the proper Bragg angle so that it can be diffracted into the focal spot at the detector. This Bragg angle is adjusted by either adjusting the radial distance of the crystal from the center of the lens or by changing the angle of the surface that is attached to the flat plate, relative to the crystalline planes. When the source is $100 \mathrm{~cm}$ from the lens, a change in the radial position of the crystal by $0.10 \mathrm{~mm}$ ( 0.0040 inches) will change the Bragg angle by 20 arc sec. When the same set of crystalline planes is used for two adjacent rings, one ring is displaced radially outward by $2 \mathrm{~mm}$ and the other ring is displaced radially inward by $2 \mathrm{~mm}$ from the nominal radius for that set of crystalline planes. To compensate for this change in radius, the angle of the crystalline planes relative to the mounting surface will be increased by 400 arc sec for one of the rings and decreased by $400 \mathrm{arc} \mathrm{sec}$ for the other ring. Thus the gamma rays will be focused slightly closer to the lens for one of the rings and slightly farther away for the other ring. If the detector is placed half way between these two focus points, the focal spot will be a small ring rather than a small disk. The mean diameter of the ring will be $8 \mathrm{~mm}$. Both the finite size of the source and the crystal elements will increase this diameter. The width of the mosaic structure of the crystals will also increase this diameter as will imperfections in the alignment of the crystals. If only one ring uses a particular set of crystalline planes and alignment of the crystals is near perfect, then 90 percent of the radiation will be contained in a focal spot with a dia. between $4 \mathrm{~mm}$ and $8 \mathrm{~mm}$, depending whether the mosaic structure width is small or large, respectively. The present lens system, using 7 rings, needs an aperature in front of the NaI detector that is at least $14 \mathrm{~mm}$ in diameter to detect 90 percent of the diffracted radiation and 18 $\mathrm{mm}$ to detect 95 percent of the diffracted radiation.

Both the flatness of the mounting plate and the accuracy of cutting the crystals affects the alignment of the crystals and thus the incident Bragg angle. The design requirement set for the lens for the sum of these errors was $+/-50$ arc sec. This magnitude of error would require a change in the radial position of the individual crystals of $+1-0.25 \mathrm{~mm}$. This would result in a maximum overlap of adjacent rings of $0.5 \mathrm{~mm}$. Thus, some of the diffracted beam of gamma rays would be both diffracted and absorbed by two crystals rather that one crystal If the crystal thickness was optimized, this overlap would decrease the intensity of the diffracted beam. We expected to experience difficulties in cutting the crystals and adjusting the angle of the mounting surface, but we did not expect the difficulty we encountered in producing a mounting plate that was flat to $+/-20$ arc sec. One could grind and polish a plate to a few wavelengths of light, but when the plate was removed from its polishing base, it would warp, sometimes as much as $+/-200$ arc sec. A number of different low- $\mathrm{Z}$ materials were tried: aluminum, fused quartz, $\mathrm{SiC}$, fiber glass, and Lucite. After many tries, one plate of aluminum and one plate of fused quartz were obtained that met these minimum specifications.

The original design for the crystal diffraction lens used eight concentric rings of crystals, in which each ring used a different set of crystalline planes. At the end of the first year, the design was changed and the development of the 13-ring model was started. The actual construction of the present lens was started in June of 1999. As of the preparation of this paper, the first seven rings were completed. Rings $1,3,5$, and 7 were mounted on the aluminum plate, and rings 2,4 , and 6 were mounted on the fused quartz plate. Figure 4 shows the four rings mounted on the aluminum plate from slightly above the plane of the lens, and Figure 5 shows the three rings mounted on the quartz plate as seen from directly above the lens. A disk of lead is placed in the center of the lens to block any direct radiation from reaching the detector. 


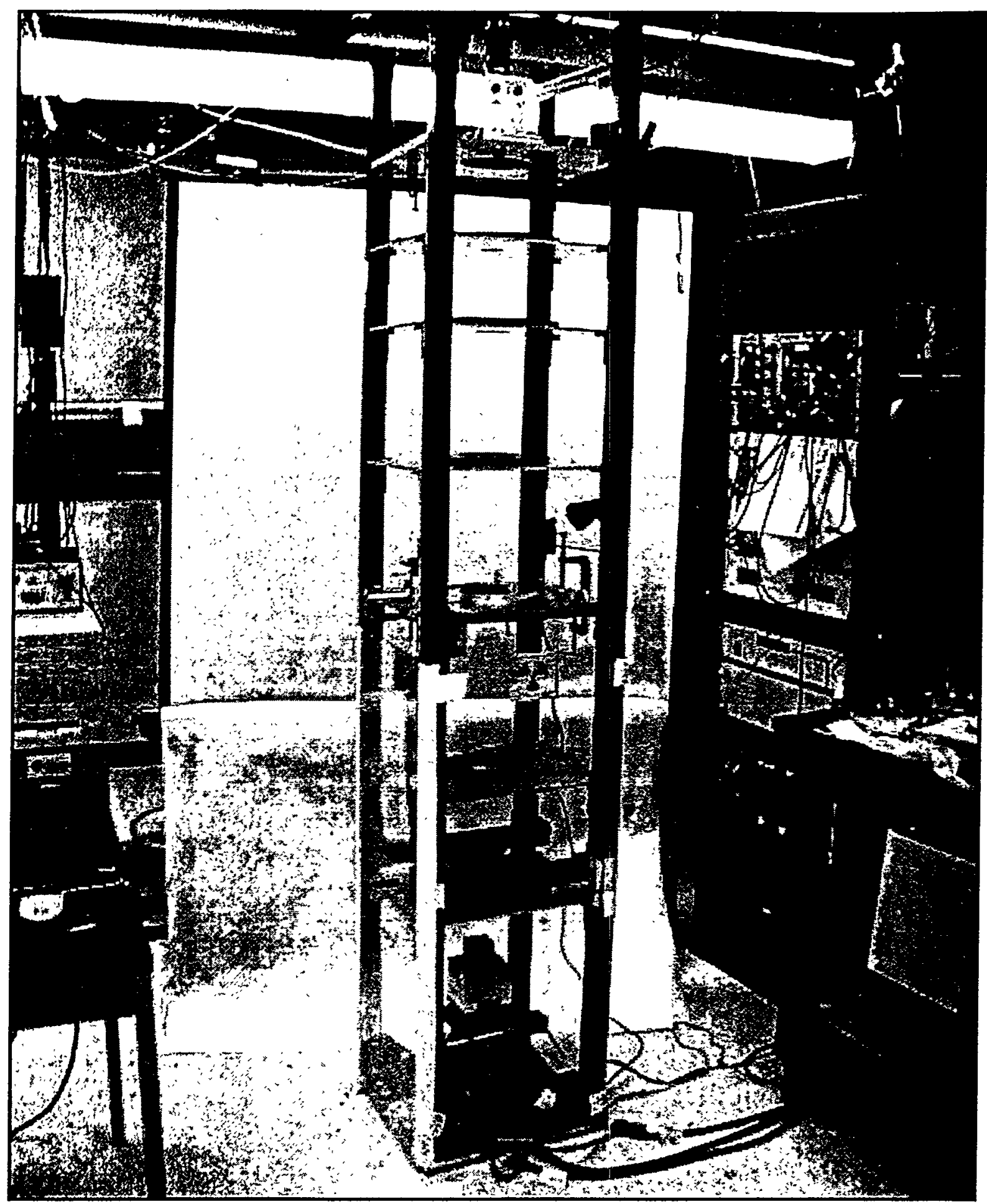

FIGURE 6. The Laue crystal lens test facility. The source is at the bottom of the picture, the lens in the middle and the deteclor at the top. 


\section{TEST RESULTS}

The lens was constructed and tested using the test stand shown in Figure 6. $A{ }^{57}$ Co source is mounted at the bottom of the test stand. This source can be moved in the two horizontal directions, $x, y$, and in the vertical direction, $z$, through a computer-controlled system. The lens is located in the middle of the test stand, just above the radiation sign. The $\mathrm{NaI}$ detector is mounted at the top of the stand. There are two collimator disks below the lens and three collimator disks above the lens. The openings in these disks are designed to allow all of the diffracted gamma rays to reach the detector but not any direct radiation from the source as the source is moved off axis. There is also a shutter just above the first collimator disk to close off the gamma ray beam, which is used for background checks. The NaI detector is $2.5 \mathrm{~cm}$ in diameter and $2.5 \mathrm{~cm}$ thick. A $2.5-\mathrm{cm}$-diameter aperture is placed directly in front of the detector.

A 3-D map of the response function of the lens with seven rings, as a function of the off-axis distance of the source, in $\mathrm{mm}$, is shown in Figure 7. The source is a $4.3-\mathrm{mm}$-diameter disk of ${ }^{57} \mathrm{Co}$ suspended in resin. The peak intensity is 65600 counts in $20 \mathrm{sec}(3780$ counts / sec), and the background is 44 counts in $20 \mathrm{sec}$ ( 2.2 counts / sec). About one half of this background is due to scattering in the lens. When one reduces the intensity of the source to what one expects in the human body, then this component of the background becomes negligible. This background will be reduced by a factor of 2 , to become 1.1 counts per sec. Under these conditions, the background scales with its volume. If one uses a more appropriate size $\mathrm{NaI}$ detector with a diameter of $18 \mathrm{~mm}$ and a thickness of $11 \mathrm{~mm}$, one can reduce the size of the detector by $(2.80 / 12.9)=0.217$ or a factor of 4.6 . When this is combined with the factor of 2 mentioned above, the reduction factor is 9.2. This reduction in detector size still maintains 95 percent of the signal.

The low intensity at the edges of the plot is part of the diffraction process. The background is much less than this count rate in the wings. This peak structure of the response function can be fit with a Lorentzian shape. This response shape is normal for a Laue lens with circular symmetry and small source size. The FWHM of the peak is $8.3 \mathrm{~mm}$. The points are taken in $1 \mathrm{~mm}$ steps in both the $\mathrm{x}$ and the $\mathrm{y}$ directions. The best FWHM that one could expect to obtain with perfect alignment of the $4 \mathrm{~mm} \mathrm{x} 4 \mathrm{~mm}$ crystals and the 4.3-mm-diameter source is $6 \mathrm{~mm}$. The efficiency of the lens changes much more slowly as one moves the source along the axis of the lens. Figure 8 shows a 3-D plot of the calculated diffraction efficiency of the original 8-ring lens design with $4 \times 4 \mathrm{~mm}$ crystals and a 4-mm-diameter source, as a function of the $\mathrm{z}$ distance along the central axis and the $x$ distance off axis. The FWHM in the $x$ direction is $6 \mathrm{~mm}$, while the FWHM in the $\mathrm{z}$ direction is about $50 \mathrm{~mm}$.

The calculated peak efficiency of 2.9 percent for the original 8-ring lens design matches the measured efficiency of 3.0 percent for the 7-ring lens. The peak efficiency is defined as the number of photons that are focused on the detector, divided by the number of photons incident on the lens. This definition includes the effect of the absorption of the gamma rays in the $\mathrm{Cu}$ crystals. This absorption is of the order of 50 percent. The double peak shape in the $\mathrm{x}$ direction, when the source is displaced along the $\mathrm{z}$ direction, is a real artifact of the diffraction process and has been observed in tests of the large Laue crystal diffraction lens built for focusing high-energy ( 200 to $800 \mathrm{keV}$ ) gamma rays.'

\section{CONCLUSIONS AND FUTURE PLANS.}

As of the preparation of this paper, the medical imaging lens consisted of seven concentric rings of crystals with a diffraction efficiency of 3 percent. The first seven rings use the following crystalline planes:[ 111$]$, [111], [200], [220], [220], [222], and [222]. The remaining six rings to be completed will use the following crystalline planes: [400], [420], [420], [422], [333], and [440]. Although only 7 of the 13 rings were completed when this paper was written, there is sufficient data to estimate the performance of the full lens. The first 7 rings of the lens perform better than the estimates made for the original 8-ring lens design. Two special features of the lens are that its efficiency does not fall off as the source becomes very small, as it does in many of the present imaging systems, and second, its ability to locate and define 


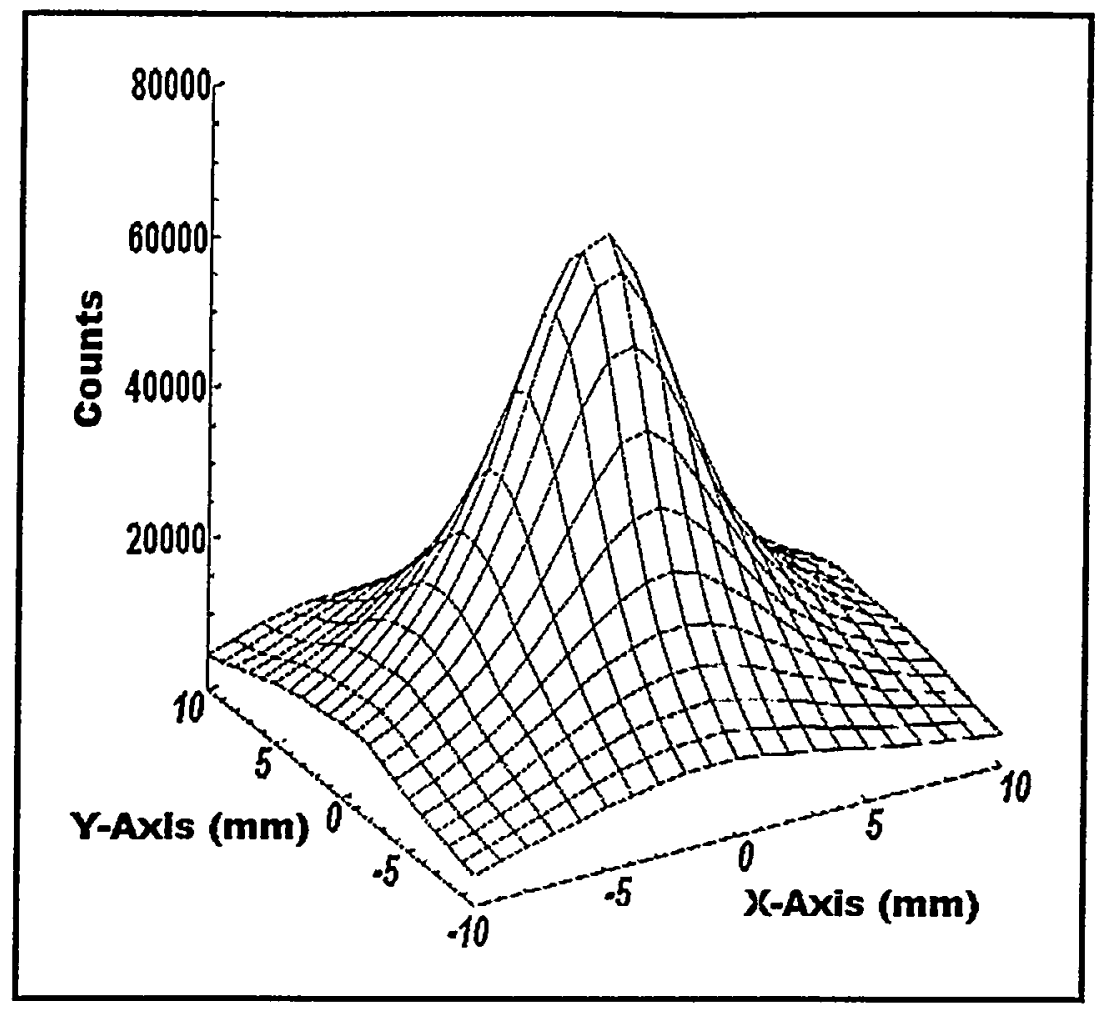

FIGURE 7. A 3-D plot of the response function of the 7-ring lens as a function of displacement of the source in the $\mathrm{x}$ and $\mathrm{y}$ directions perpendicular to the axis of the lens.

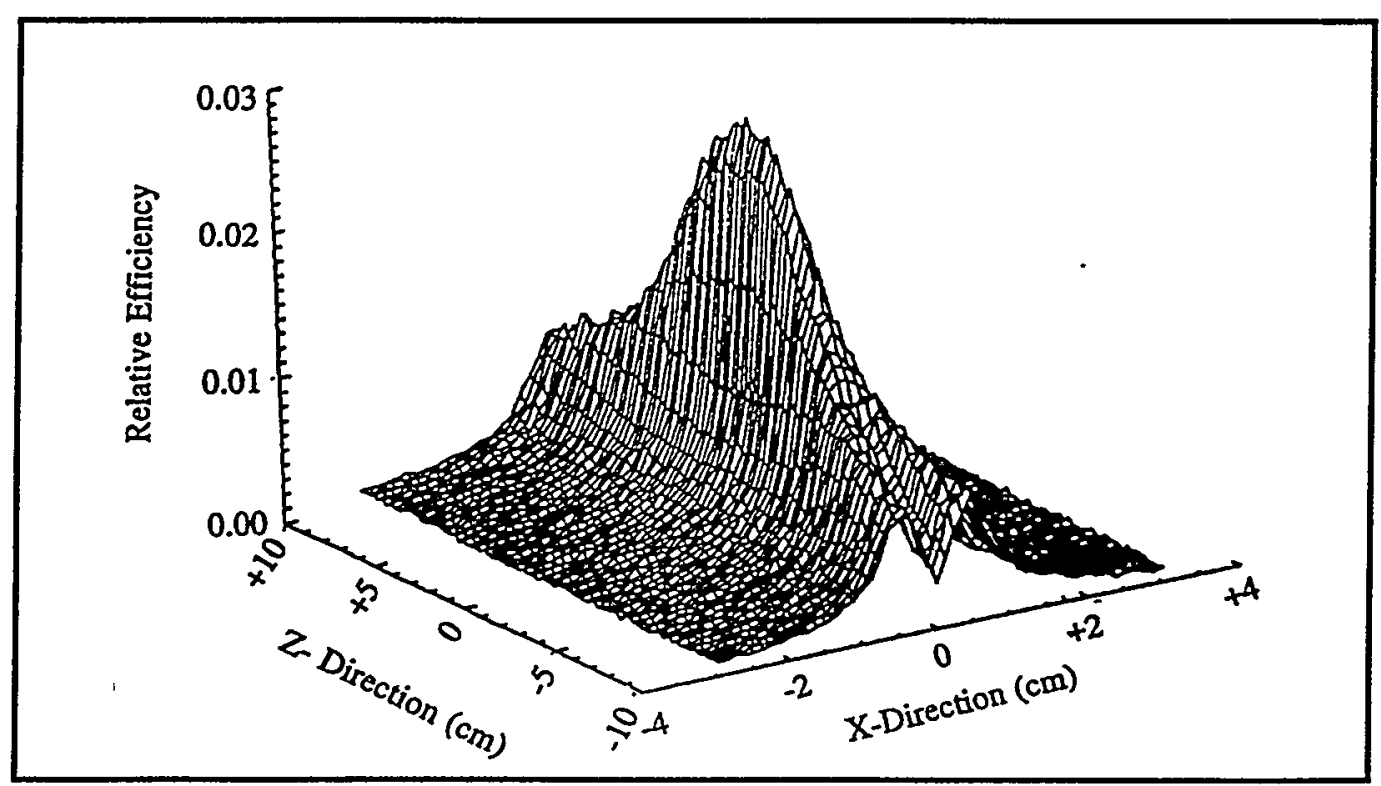

FIGURE 8. A 3-D plot of the calculated diffraction efficiency of a single Laue lens imaging system as a function of the displacement of the source in the $x$-direction of the lens and in the z-direction parallel to the axis of the lens. This plot illustrates the narrow shape of the focal volume in the $\mathrm{x}$-direction and extended shape in the $\mathrm{z}$-direction. 
the size of the tumor with mm resolution. Also of importance is the simplicity of the data analysis. There are no coincidence requirements between detectors. The signal is the sum of the individual count rates in the individual detectors. When the system is used in a scanning mode to determine the size and position of the tumor, the change in the counting rate in each detector will also be recorded. This will indicate in which direction the count rate is increasing or decreasing.

The overall efficiency of a single lens system is the product of the diffraction efficiency times the solid angle of the lens divided by $4 \pi$. For a single lens system with all 13 concentric rings of crystals and diffraction efficiency of 3 percent, the overall efficiency is approximately $0.03 \times\left\{155 \mathrm{~cm}^{2} /\left(100^{2} \times 4 \pi\right)\right\}$ $=0.03 \times 1.23 \times 10^{-3}=3.7 \times 10^{-5}$. The full 6-lens imaging system is calculated to have a overall efficiency of $2.2 \times 10^{-4}$. This is comparable to the efficiencies of present day scanners, $3 \times 10^{-4}$ for large sources. $^{7}$ The full body scanners have lower overall efficiencies of the order of $3 \times 10^{-5}$ for small sources. ${ }^{7}$ The crystal lens system, however, has a lower background ${ }^{2,3,7}$ and maintains the same over-all efficiency of $3 \times 10^{-4}$ for small sources $(10 \mathrm{~mm}-1 \mathrm{~mm})$, thus, it has a much higher sensitivity for small sources. The lower background comes partly from the fact that the phototubes in the full body scanner look at 25 times the volume $\mathrm{NaI}$ that is viewed by the phototube in the lens system and partly because the full body scanner has aditional background associated with scattering of the gamma rays in the body. The lens system in insensitive to this component. As the source size is inceased above $20 \mathrm{~mm}$, the increase in counting rate in the lens system increases proportionally to the radius as compared to the rate in a detector that looks directly at the source, where the increase in counting rate increases as the square of the radius. With this large a tumor, both the lens system and the collimator system can see the tumor with ease. The lens system is still useful for these larger tumors in that it can resolve the 3-D shape of the tumor with mm resolution.

Just how much radioactivity is taken up by the tumor varies from case to case. Organs with a high blood content, like the heart muscle, retain about 2.2 micro-Curies per cc of muscle. ${ }^{7}$ The typical cancer retains about 6 times this amount, about 13 micro-Curies per cc. ${ }^{7}$ Using these numbers, the full 6-lens imaging system is expected to have a count rate of about 100 counts per sec with a background of 1.5 counts per $\mathrm{sec}$ for a $1 \mathrm{cc}$ volume tumor. Thus a tumor of only $0.1 \mathrm{cc}$ would still give an easily detectable signal of 10 counts per sec. Typically, the absorption of the gamma rays in the body will reduce these numbers by a factor of 2. A volume of $1 \mathrm{cc}$ corresponds to a $12.4-\mathrm{mm}$-dia. sphere. A $0.1 \mathrm{cc}$ volume corresponds to a sphere $2.9 \mathrm{~mm}$ in radius. The present full body scanners have difficulty seeing tumors that have a volume of less than $1 \mathrm{cc}^{7}$ while this lens system should be able to see a $0.1 \mathrm{cc}$ tumor. The crystal lens system is not meant to replace the full body scanner. The full body scanner looks at a large area (700 to $\left.1000 \mathrm{~cm}^{2}\right)$ at one time, while the lens systam looks at only $1 \mathrm{~cm}^{2}$ with high sensitivity. Thus, it would take a long time to scan the full body. A scan of a women's breast woild be possible, for example. In most cases one would use the lens system to supplement rather than replace the full body scanner, by confirming the existence of a tumor detected by the full body scanner and measuring its size and position with $\mathrm{mm}$ accuracy.

\section{ACKNOWLEDGMENTS}

The authors would like to thank Robert N. Beck and Nicholas J. Yasillo for their comments and advice and medical data needed to evaluate the proposed crystal diffraction medical imaging system, Ron Martin and Bart Claire for their contributions to this project in its early stages, Algirdas Paugys for his assistance in constructing the crystal diffraction lenses and the test stand that was used to assemble and test the lens system, Frank Bittles and Ruben Khachatryan of the APS optics shop and Dan Brown and Michael Polowinczak of the ANL machine shop for their assistance in cutting the copper crystals and making the flat plates used to support the crystals in the lens, and Bernard Hamelin and his crystal growing group at ILL, in Grenoble for furnishing the two large boules of copper single crystals, which were used to cut the 
small, $4 \mathrm{~mm} \times 4 \mathrm{~mm}$, crystal elements used in the lens. This work was supported by the U.S.

Department of Energy, Basic Energy Sciences, under contract no. W-31-109-Eng-38.

\section{REFERENCES}

1. R. H. Wagner, S. M. Karesh and J. R. Halama, Questions and Answers in Nuclear Medicine, Chapter 4, Mosby, St. Louis, 1999.

2. J. B. Svedberg, "Image Quality of Gamma Camera System," Phys. Med. Biol., 13, PP 597-610, 1968.

3. R. N. Beck, M. W. Schuh, T. D. Cohen, and N. Lembares, "Effects of Scattered Radiation on Scintillation Detector Response," in Medical Radioisotope Scintigraphy, I, pp 595-616, IAEA, Vienna, 1969.

4. R. Wicks M. Blau, "Effect of Distortion on Anger Camera Field-Uniformity Correction: Concise Communication," J. Nucl. Med., 20, pp 252-254, 1979.

5. P. J. Peller, N. Y. Khedkar, and C. J. Martinez, "Breast Tumor Scintigraphy," J. Nucl. Med. Technol, 24, pp 198-203, 1996.

6. H. Wang, C. Scarfone, K. L. Greer, R. E. Coleman, R. J. Jaszczak, "Prone Breast Tumor Imaging Using Vertical Axis-of-Rotation (VAOR) SPECT Systems," IEEE Transactions on Nucl. Med. Science 44, pp 1271-1276, 1997.

7. Communications from Nicholas Yasillo, R. N. Beck and P. La Riveiere, based on clinical studies performed by the Radiological Department of the University of Chicago.

8. R. K. Smither, “3-Dimensional'Imaging System Using Crystal Diffraction Lenses,” U.S. Patent No. 5,869,841, issued to R. K. Smither on Feb. 9, 1999.

9. R. K. Smither, P. von Ballmoos, P. B. Fernandez, T. Graber, J. Naya, F. Albernhe, and G. Vedrenne, "Review of Crystal Diffraction and Its Application to Focusing Energetic Gamma Rays", Experimental Astronomy 6, pp 47-56, 1995. 